\title{
Glossopharyngeal Nerve Transection Eliminates Quinine-Stimulated Fos-Like Immunoreactivity in the Nucleus of the Solitary Tract: Implications for a Functional Topography of Gustatory Nerve Input in Rats
}

\author{
Camille T. King, ${ }^{1}$ Susan P. Travers, ${ }^{2}$ Neil E. Rowland, ${ }^{1}$ Mircea Garcea, ${ }^{1}$ and Alan C. Spector ${ }^{1}$ \\ ${ }^{1}$ Department of Psychology, University of Florida, Gainesville, Florida 32611, and 2Department of Oral Biology, College of \\ Dentistry, Ohio State University, Columbus, Ohio 43210
}

The relationship between specific gustatory nerve activity and central patterns of taste-evoked neuronal activation is poorly understood. To address this issue within the first central synaptic relay in the gustatory system, we examined the distribution of neurons in the nucleus of the solitary tract (NST) activated by the intraoral infusion of quinine using Fos immunohistochemistry in rats with bilateral transection of the chorda tympani (CTX), bilateral transection of the glossopharyngeal nerve (GLX), or combined neurotomy (DBLX). Compared with nonstimulated and water-stimulated controls, quinine evoked significantly more Fos-like-immunoreactive (FLI) neurons across the rostrocaudal extent of the gustatory NST (gNST), especially within its dorsomedial portion (subfield 5). Although the somatosensory aspects of fluid stimulation contributed to the observed increase in FLI neurons, the elevated number and spatial distribution of FLI neurons in response to quinine were remarkably distinguishable from those in response to water. GLX and DBLX produced a dramatic attenuation of quinine-evoked FLI neurons and a shift in their spatial distribution such that their number and pattern were indiscernable from those observed in water-stimulated controls. Although CTX had no effect on the number of quinine-evoked FLI neurons within subfield 5 at intermediate levels of the gNST, it produced intermediate effects elsewhere; yet, the spatial distribution of the quinine-evoked FLI neurons was not altered by CTX. These findings suggest that the GL provides input to all FLI neurons responsive to quinine, however, some degree of convergence with CT input apparently occurs in this subpopulation of neurons. Although the role of these FLI neurons in taste-guided behavioral responses to quinine remains speculative, their possible function in oromotor reflex control is considered.

Key words: taste; nucleus of the solitary tract; Fos immunohistochemistry; nerve transection; topography; glossopharyngeal nerve; chorda tympani nerve
In the auditory, visual, and somatosensory systems, the central projections of first order neurons represent their respective sensory receptor surfaces in a topographic manner. This anatomical organization is often preserved and elaborated at higher levels of the sensory pathway and appears to be a fundamental architecture related to stimulus processing. The immunohistochemical detection of Fos [Fos-like immunoreactivity (FLI)], the protein product of the immediate early gene $c$-fos, has effectively served to identify with cellular resolution, populations of CNS neurons that have been activated by sensory stimulation (Sagar et al., 1988; Morgan and Curran, 1989; Sheng and Greenberg, 1990; Sharp et al., 1993). In recent years, the localization of FLI has supported the existence of functional topographies in the CNS, such as tonotopy in the auditory system (Ehret and Fischer, 1991; Friauf, 1992, 1995), somatotopy in cortical barrels in rodents

\footnotetext{
Received Oct. 13, 1998; revised Dec. 21, 1998; accepted Jan. 26, 1999.

This work was supported in part by Grants R01-DC01628 (A.C.S.) and R01DC00416 (S.P.T.) from the National Institute on Deafness and Other Communication Disorders. Alan C. Spector is the recipient of a Research Career Development Award (K04-DC00104) from the National Institute on Deafness and Other Communication Disorders. We thank Orenda Lyons, Nick Guagliardo, Suzanne Sealey, and Kim Robertson for technical assistance and Dr. M. S. King for comments on an earlier version of this manuscript.

Correspondence should be addressed to Dr. Alan C. Spector, Department of Psychology, University of Florida, Gainesville, FL 32611-2250.

Parts of this paper were presented at the Twelfth International Symposium on Olfaction and Taste, San Diego, CA, July, 1997.

Copyright (C) 1999 Society for Neuroscience $\quad 0270-6474 / 99 / 193107-15 \$ 05.00 / 0$
}

(Mack and Mack, 1992; Steiner and Gerfan, 1994; Melzer and Steiner, 1997), and eye-related columns in visual cortex (Beaver et al., 1993; Mitchell et al., 1995; Montero and Jian, 1995).

Exploiting this strategy in the gustatory system of rats, Harrer and Travers (1996) detected an interesting chemotopy using quinine, a compound described as bitter by humans, and sucrose ("sweet" to humans) as taste stimuli. After quinine stimulation, FLI neurons were concentrated medially within the rostral portion of the nucleus of the solitary tract (NST), the first central synaptic relay in the gustatory system. After stimulation with sucrose, FLI neurons were distributed more evenly along its mediolateral axis. Although the more medial distribution of FLI neurons after quinine stimulation is consistent with the more medial distribution of IXth nerve afferent fibers compared with VIIth nerve fibers, the chemotopic organization suggested by the FLI data does not correspond entirely with the known orotopic arrangement of primary gustatory nerve terminations in the NST. For example, based on the response properties of the gustatory nerves and their corresponding central terminations (Table 1), sucrose stimulation should have resulted in increased FLI at more rostral levels of the NST compared with quinine stimulation, but this outcome was not statistically realized. Thus, the chemotopy reflected by FLI was not fully presaged by the terminal distributions of the respective gustatory nerves.

There are some important caveats to consider with respect to these findings. Because the animals potentially swallowed at least 


\begin{tabular}{|c|c|c|c|c|}
\hline Gustatory nerve & Oral cavity innervation & $\begin{array}{l}\text { Terminal field } \\
\text { location in } \mathrm{rNST}^{a}\end{array}$ & "Best" stimulus ${ }^{b}$ & Reference \\
\hline $\begin{array}{l}\text { Chorda tympani branch } \\
\text { of V IIth nerve }(\mathrm{CT})\end{array}$ & Anterior tongue $^{1}$ & Rostral Pole & Sodium salts and acids ${ }^{2}$ & $\begin{array}{l}{ }^{1} \text { Beidler, 1969; }{ }^{2} \text { Pfaffmann, 1955; Frank } \\
\quad \text { et al., } 1983\end{array}$ \\
\hline $\begin{array}{l}\text { Greater superficial branch } \\
\text { of VIIth nerve (GSP) }\end{array}$ & Soft palate ${ }^{1}$ & Rostral Pole & Sugars $^{2}$ & $\begin{array}{l}{ }^{1} \text { Cleaton-Jones, } 1976 ;{ }^{2} \text { Nejad, 1986; } \\
\text { Harada et al. } 1997\end{array}$ \\
\hline $\begin{array}{l}\text { Lingual-tonsilar branch of } \\
\text { IXth nerve (GL) }\end{array}$ & Posterior tongue $^{1}$ & Caudal rNST & Alkaloids and acids ${ }^{2}$ & $\begin{array}{l}{ }^{1} \text { Guth, 1957; }{ }^{2} \text { Yamada, 1966; Frank, 1991; } \\
\text { Dahl et al., } 1997\end{array}$ \\
\hline $\begin{array}{l}\text { Superior laryngeal branch } \\
\text { of Xth nerve (SL) }\end{array}$ & Laryngeal epithelium ${ }^{1}$ & $\begin{array}{c}\text { Caudal rNST \& } \\
\text { caudal NST }\end{array}$ & Water $^{2}$ & $\begin{array}{l}{ }^{1} \text { Miller, 1977; Travers and Nicklas, 1990; } \\
{ }^{2} \text { Andrew, 1956; Shingai, } 1980\end{array}$ \\
\hline
\end{tabular}

${ }^{a}$ Hamilton and Norgren (1984); rNST denotes gustatory portion of the NST and represents locus of the greatest density of terminals.

$b$ "Best Stimulus" refers to the chemicals to which each nerve is the most electrophysiologically responsive relative to other stimuli.

some amount of the stimuli (at high concentrations) over a $30 \mathrm{~min}$ period, postingestive factors rather than gustatory events may have contributed to the $c$-fos response in the NST. Moreover, although both quinine and sucrose led to significantly enhanced FLI in the NST compared with no stimulation, the gustatoryelicited increases in FLI did not reach statistical significance relative to water. Consequently, whether the observed patterns of FLI have a gustatory origin remains to be clarified.

Given that quinine and sucrose produce distinct patterns of neural activity in the NST and in perhaps other regions of the gustatory neuroaxis, what is the relative contribution of the various sources of peripheral input to the respective topographies? At issue is how taste input derived from specific gustatory nerves innervating specific oral receptor fields is channeled throughout the brain. One strategy to approach this problem is to examine if and how patterns of taste-stimulated FLI are altered by the selective removal of input from gustatory nerves. We therefore examined the number and distribution of FLI neurons elicited by intraoral infusion of quinine in rats with bilateral transection of the GL, CT, or combined neurotomy. Accordingly, the relative contribution of these targeted gustatory nerves to the quinine pattern was assessed, while confirming that the quininestimulated FLI has an orosensory origin.

\section{MATERIALS AND METHODS}

Subjects. Forty-one naive male Sprague Dawley rats (Charles River Laboratories, Wilmington, MA) weighing between 250 and $275 \mathrm{gm}$ at the start of the experiment served as subjects. The rats were individually housed in hanging wire mesh cages where light cycle (lights on 6 A.M. to 6 P.M.), temperature, and humidity were automatically controlled. All manipulations were performed during the light phase. Purina chow (5001; Ralston-Purina, St Louis, MO) and water were available ad libitum.

Surgical procedures. Rats were deeply anesthetized (intramuscularly) with a mixture of ketamine hydrochloride $(125 \mathrm{mg} / \mathrm{kg})$ and xylazine hydrochloride $(5 \mathrm{mg} / \mathrm{kg}$ ) and subjected to bilateral transection of the CT, the GL, both the CT and GL, or sham surgery. For details of surgical procedures, refer to St. John et al. (1994). Briefly, for chorda typmani transection (CTX), the external auditory meatus was widened, and the tympanic membrane and ossicles were removed to reveal the CT, which was then avulsed with forceps. For glossopharyngeal nerve transection (GLX), the sublingual and submaxillary salivary glands and the sternohyoid, omohyoid, and posterior belly of the digastric muscles were retracted, and the fascia underlying the hypoglossal nerve was carefully dissected to reveal the GL near the external medial wall of the bulla. A 2-3 $\mathrm{mm}$ portion of the GL was cut and removed. The incision was closed with sutures. Combined bilateral neurotomies (DBLX) were performed by transecting both the CT and GL in the same subject. In SHAM animals, the GL was exposed as described above but was not disturbed, and the tympanic membrane was punctured.

During the same surgical session each rat was implanted bilaterally with intraoral cannulae through which infusions of stimuli directly into the oral cavity could be achieved (Grill and Norgren, 1978). The cannula consisted of a $35 \mathrm{~mm}$ length of polyethylene tubing (PE-100) heat-flared at one end to support a small Teflon washer that was threaded onto the tubing. The PE tubing was placed just lateral to the first maxillary molar and at its exit, just lateral to the skull, it was attached to a $16 \mathrm{~mm}$ length of 19 gauge stainless steel tubing. Cannulae were secured to the skull with dental acrylic and screws. A topical antibiotic was applied, and wounds were sutured closed. For the first 3 postoperative days, rats were given subcutaneous injections of penicillin (30,000 units) to prevent infection. Wet mash [powdered Purina chow mixed with water and supplemented with Nutri-Cal (Evsco Pharmaceuticals, Buena, NJ)] was available ad libitum for 5-7 days to promote feeding. The cannulae were cleaned daily to maintain patency and prevent infection. After 14-17 d of recovery, behavioral procedures were initiated.

Stimulus delivery. All animals were at least $85 \%$ of their presurgical body weights before testing. One CTX animal lost excessive body weight after surgery and was therefore excluded from the experiment and euthanized. SHAM and nerve-transected rats were assigned to one of three stimulus conditions: no fluid stimulus (NS), $0.003 \mathrm{M}$ quinine hydrochloride (Q), or distilled water (W). This assignment indicated the stimulus condition on the test day.

Behavioral procedures were based on those described by Harrer and Travers (1996). Rats were habituated and tested in a cylindrical Plexiglas chamber connected via PE tubing to a syringe inf usion pump (infusion pump 22; Harvard Apparatus, South Natick, MA). The chamber and pump offered the advantage of delivering stimuli at a constant rate without the experimenter having to be present in the testing room. Because stress has been shown to produce "nonspecific" Fos elevation (Dragunow and Faull, 1989), subjects were familiarized with the chamber for 3 consecutive days before testing in an effort to reduce the stress associated with novelty. During these sessions, the animal's left cannula was attached via PE tubing to the syringe on the infusion pump, and the animal was placed in the chamber for $1 \mathrm{hr}$. After this adaptation period, the experimenter re-entered the room to start the inf usion pump, which delivered $7 \mathrm{ml}$ of distilled water over $30 \mathrm{~min}(0.233 \mathrm{ml} / \mathrm{min})$. This inf usion period allowed the subjects assigned to the $\mathrm{W}$ or the $\mathrm{Q}$ group to adapt to the intraoral infusion of fluid. At the end of $30 \mathrm{~min}$, the experimenter re-entered the room to stop the pump. Animals assigned to the NS group never received fluid stimulation; however, to maintain consistency, the experimenter did enter the testing room at the start and finish of the $30 \mathrm{~min}$ infusion period. The animal remained in the chamber for $45 \mathrm{~min}$ before being returned to its home cage.

On the test day, the behavioral protocol was identical to that followed during the 3 previous habituation days except that during the infusion period, animals assigned to the $\mathrm{Q}$ group received $0.003 \mathrm{M}$ quinine hydrochloride in place of distilled water.

Brain histology. Immediately after the 45 min post-infusion period on the test day, animals were deeply anesthetized with an overdose of sodium pentobarbital and perfused intracardially with heparinized 0.15 $\mathrm{M} \mathrm{NaCl}$, followed by sodium phosphate-buffered $4 \%$ paraformaldehyde, $\mathrm{pH}$ 7.3. Brains were removed and post-fixed overnight at $4^{\circ} \mathrm{C}$. The medulla, pons, and portions of the forebrain containing the piriform cortex were cut in the coronal plane $(75 \mu \mathrm{m})$ using a vibratome. Every other section was processed for Fos immunoreactivity. These sections were pretreated with sodium borohydride $[1 \%$ in potassium PBS 
(KPBS)] for $20 \mathrm{~min}$, rinsed in KPBS, and then incubated with Fos antibody (lot number A055; Santa Cruz Biotechnology, Santa Cruz, CA) at a dilution of $1: 10,000$ in $0.4 \%$ Triton X-100 in KPBS for $72 \mathrm{hr}$ at $4^{\circ} \mathrm{C}$. The sections were then rinsed with KPBS, placed for $4 \mathrm{hr}$ in biotinylated goat anti-rabbit IgG (Zymed, San Francisco, CA) at a dilution of 1:600 at room temperature, rinsed with KPBS, and then placed at $4^{\circ} \mathrm{C}$ in avidinbiotinylated peroxidase complex (ABC kit; Vector Laboratories, Burlingame, CA) overnight. Finally, the sections were rinsed in KPBS and placed in sodium phosphate buffer containing $0.03 \%$ diaminobenzidine, $0.008 \%$ nickel ammonium sulfate, $0.008 \%$ cobalt chloride, and $0.0075 \%$ hydrogen peroxide. Stained sections were mounted on chrome-alumcoated slides, dehydrated, and coverslipped. Alternate sections for 12 of the brains (SHAM-Q, $n=2$; SHAM-W, $n=2$; SHAM-NS, $n=1$; CTX-Q, $n=2$; GLX-Q, $n=2$; DBLX-Q, $n=2$; DBLX-NS, $n=1$ ) were stained with $0.1 \%$ thionin, mounted on chrome-alum-coated slides, dehydrated, and coverslipped. These sections were used to delineate anatomical subdivisions within the rostral NST. The alternate sections for the remaining brains could not be used for histological analysis.

Microscopic analysis. The tracing and counting of FLI-positive cells were performed by an experimenter who was unaware of either the surgical group or stimulus condition to which the subject was assigned. Sections were observed under $4-40 \times$ objectives with a Nikon light microscope equipped with a video camera. This arrangement enabled the microscopic image to be displayed on a video monitor onto which transparencies could be attached. Five standard sections of the NST spaced throughout its rostrocaudal extent were selected for analysis based on the terminal fields of the gustatory nerves (Hamilton and Norgren, 1984). Four of these sections were selected from the gustatory zone of the NST (gNST), and one section was chosen posterior to this portion of the nucleus (NgNST). The rostral section of the gustatory NST (RgNST), where the CT chiefly terminates, was taken at the caudal level of the dorsal cochlear nucleus (cDCN). Two sections were chosen from the intermediate portion of the gNST where the GL principally terminates but has some, albeit slight, overlap with $\mathrm{CT}$ primary afferent fibers. The intermediate rostral section (IRgNST) selected was $\sim 500 \mu \mathrm{m}$ caudal to $\mathrm{cDCN}$, and the intermediate caudal section (ICgNST) selected was $\sim 900 \mu \mathrm{m}$ caudal to cDCN $(\sim 150-300 \mu \mathrm{m}$ rostral to the level at which the NST first abuts the fourth ventricle). The caudal gustatory section (CgNST) was chosen at the level where the posterior aspect of the GL terminal field would be located, $\sim 1500 \mu \mathrm{m}$ caudal to $\mathrm{cDCN}(\sim 225-375$ $\mu \mathrm{m}$ caudal to the level at which the NST first abuts the fourth ventricle). The nongustatory section (NgNST) selected was at the level of the anterior aspect of the area postrema, $\sim 2000 \mu \mathrm{m}$ caudal to cDCN.

All FLI-positive nuclei within the borders of the NST in each section were traced from the video monitor onto the overlaid transparency. Because considerable differences in staining intensities among neurons were observed, FLI neurons were classified as exhibiting light, intermediate, or dark staining relative to other neurons within the same brain. For example, neurons in the piriform cortex consistently showed strong FLI under all conditions and were therefore examined beforehand to assure that each brain was successfully processed for FLI and to provide an index of staining intensity. "Dark" staining neurons were typically very dark brown to black in color. "Intermediate" staining cells ranged from dark brown to light brown in color. "Light" staining neurons were just distinguishable as being stained nuclei, a very light brown/pale color.

To objectively quantify the spatial distribution of FLI neurons within each coronal section, we parceled the traced gNST in each section into six "subfields" based on its dimensions (Fig. 1a). The gNST was first divided into three equal segments by drawing two lines perpendicular to the long axis of the nucleus. At the two points where each perpendicular line crossed the long axis, the dorsoventral axis of the NST was measured and divided by 2 . Each one-third segment of the long axis was then redrawn so that the dorsal and ventral portions were roughly equal. For the 12 brains having alternate sections stained with thionin, the gNST was parceled into anatomically defined subdivisions [medial (M), rostral central (RC), ventral (V), and rostral lateral (RL)] as previously described by Whitehead (1988) in the hamster and Halsell et al. (1996) in the rat. This was accomplished using a computer-interfaced camera lucida system and software (Lucivid/Neurolucida; Microbright, Inc.) which allowed anatomical structures to be traced onto a miniature video screen that was viewed directly through the microscope objectives, simultaneous with the section. These tracings were then enlarged and overlaid onto the corresponding transparencies containing the FLI drawings. In this manner, the distribution of FLI neurons in the gNST could
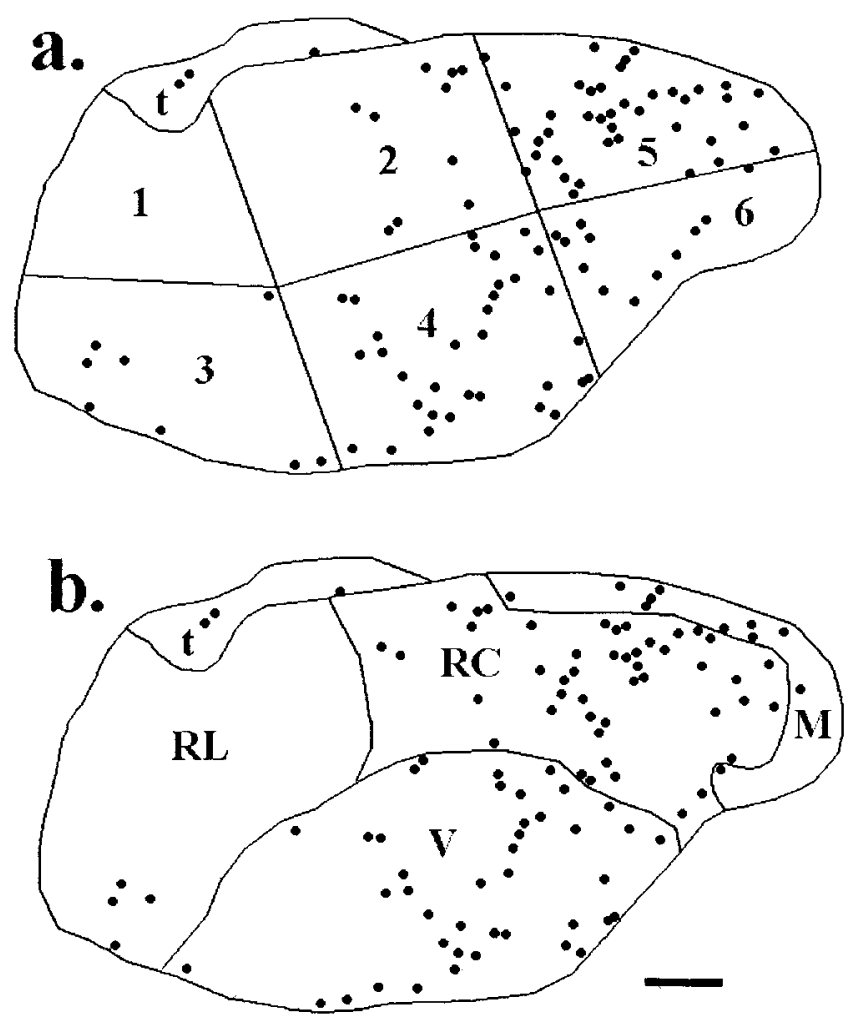

Figure 1. Identical tracings of FLI neurons found in the IRgNST section in a quinine-stimulated control rat parceled into the subfields used for the present analysis $(a)$, as described in Materials and Methods, and into previously defined anatomical subdivisions $(b)$ : medial $(M)$, rostrocentral $(R C)$, ventral $(V)$, and rostrolateral $(R L)$. These subdivisions were delineated with the aid of thionin-stained adjacent sections. Note that portions of subfields 5 and 6 in this section $(a)$ collectively capture all the FLI neurons in the medial half of the area delineated as RC in $b$, the same region in which Harrer and Travers (1996) found the majority of quinineelicited FLI neurons. $t$, Solitary tract. Scale bar, $100 \mu \mathrm{m}$.

be compared using the subfields in the present study and the previously defined anatomical subdivisions (Fig. 1).

Preplanned comparisons between SHAM-Q rats $(n=6)$ and each of the other groups [SHAM-W $(n=6)$, CTX-Q $(n=6)$, GLX-Q $(n=6)$, DBLX-Q $(n=6)$, SHAM-NS $(n=5)$, and DBLX-NS $(n=5)]$ were performed using two-tailed $t$ tests to assess significant differences in the number of FLI neurons counted, regardless of staining intensity, for Total gNST (see Fig. 5), at each rostrocaudal level of the gNST (see Fig. 6), for each subfield (see Fig. 7), and for each subfield at each rostrocaudal level (see Fig. 8). Separate one-way ANOVAs, one for each dependent variable, were then conducted using all groups to assess further effects of nerve transections on FLI neurons. If the ANOVA revealed significant differences (ANOVA results are listed in the corresponding figure legends), then post hoc Bonferroni-adjusted two-tailed $t$ tests were performed between SHAM-W rats and each of the other groups, as well as comparisons between each of the nerve-transected groups. Similar statistical analyses were performed to determine differences in the proportion of neurons in the Total gNST which were classified as light, intermediate, or dark staining.

Tongue histology. After animals were perfused, their tongues were post-fixed in $10 \%$ buffered formalin. The efficacy of CT nerve transections was verified by examination of the anterior tongues from CTX $(n=$ $6)$, DBLX $(n=11)$, and a representative sample of SHAM $(n=6)$ animals. Each anterior tongue was immersed in distilled water for $1 \mathrm{hr}$ and then stained in $0.5 \%$ methylene blue followed by a rinse in distilled water. It was then cut along the midline into right and left halves, and the underlying muscle and connective tissue were removed. The halves were flattened between two glass slides for microscopic analysis of taste pores within the fungiform papillae. A taste pore appeared as a blue dot roughly centered in a pale staining fungiform papilla. The number of 
Table 2. Tongue histological results

\begin{tabular}{llrlll} 
& \multicolumn{2}{l}{ Fungiform papillae } & & $\begin{array}{l}\text { Circumval- } \\
\text { late papilla }\end{array}$ \\
\cline { 2 - 4 } Group $(n)$ & Number & $\begin{array}{l}\text { Number } \\
\text { with a pore }\end{array}$ & $\begin{array}{l}\text { Percent } \\
\text { with a pore }\end{array}$ & $\begin{array}{l}\text { Number of } \\
\text { taste buds }\end{array}$ \\
\hline SHAM (6) & $156.7 \pm 4.4$ & $150.8 \pm 4.5$ & $96.3 \pm 0.5$ & & $391.3 \pm 19.5$ \\
CTX (6) & $131.7 \pm 6.2$ & $16.3 \pm 7.0$ & $11.5 \pm 4.6$ & \\
GLX (6) & & $16.9 \pm 3.8$ & $12.3 \pm 2.6$ & 0.0 \\
DBLX (11) & $133.4 \pm 4.1$ & 16.9 & 0.0 \\
\hline
\end{tabular}

fungiform papillae with and without an intact taste pore were counted. A one-way ANOVA followed by post hoc $t$ tests were performed to assess statistical differences in the number of taste pores between groups.

The portion of the posterior tongue containing the circumvallate papilla from each GLX $(n=6)$, each DBLX $(n=11)$, and a representative sample of SHAM $(n=6)$ animals was embedded in paraffin, sectioned at $10 \mu \mathrm{m}$ on a rotary microtome, mounted, and stained with hematoxylin and eosin. The number of taste buds within the circumvallate papillae was counted. Absence of taste buds is indicative of successful nerve transection (Guth, 1957; Ganchrow and Ganchrow, 1989).

\section{RESULTS}

\section{Tongue histology}

Examination of fungiform and circumvallate papillae confirmed the efficacy of nerve transections (Table 2). A one-way ANOVA revealed a main effect of group on the number of taste pores in the fungiform papillae $\left(F_{(2,20)}=216.27 ; p<0.0001\right)$. The post hoc tests revealed that rats in both the CTX and DBLX groups always had fewer taste pores than SHAM rats (both, $p<0.0001$ ) One CTX and one DBLX rat each had $\sim 33 \%$ of their fungiform papillae containing a taste pore, which is 3 times the mean number of pores found in rats with bilateral transection of the CT. Several factors argue against the possibility that these represented functionally "regenerated" taste buds. First, the persistence of taste buds after CT transection has been reported previously (Whitehead et al., 1987; St. John et al., 1994; see also Ganchrow and Ganchrow, 1989). Second, after CT transection, at least $28 \mathrm{~d}$ of recovery were required to support partial behavioral competence on taste-guided tasks, and $49 \mathrm{~d}$ were necessary to allow for complete behavioral recovery (St. John et al., 1995). Although partial regeneration in these two animals cannot be ruled out in the present study, it is unlikely, based on these previous findings, that $14-17 \mathrm{~d}$ of recovery would permit nerve regeneration capable of supporting function. No rat in either the GLX or DBLX group had any taste buds with taste cells in the circumvallate papillae, confirming the success of GL transections (Fig. 2, Table 2)

\section{Fos-like immunoreactivity in sham-operated rats} Number of FLI neurons

As is evident in Figures 3 and 4, the number of FLI neurons in the gNST of SHAM rats was dependent on the stimulus condition and the level of the NST examined. The mean total number of quinine-evoked FLI neurons (SHAM-Q; Fig. 4, top set of dashed lines) across the four most rostral sections of the gustatory NST (i.e., gNST) analyzed was about 6 times greater than that for nonstimulated rats (SHAM-NS; $p<0.005$ ). These results were expected based on the findings of Harrer and Travers (1996), who also demonstrated elevated FLI in quinine-infused rats compared with nonstimulated rats. In the previous study, distilled water stimulation evoked only a nonsignificant increase in FLI but in
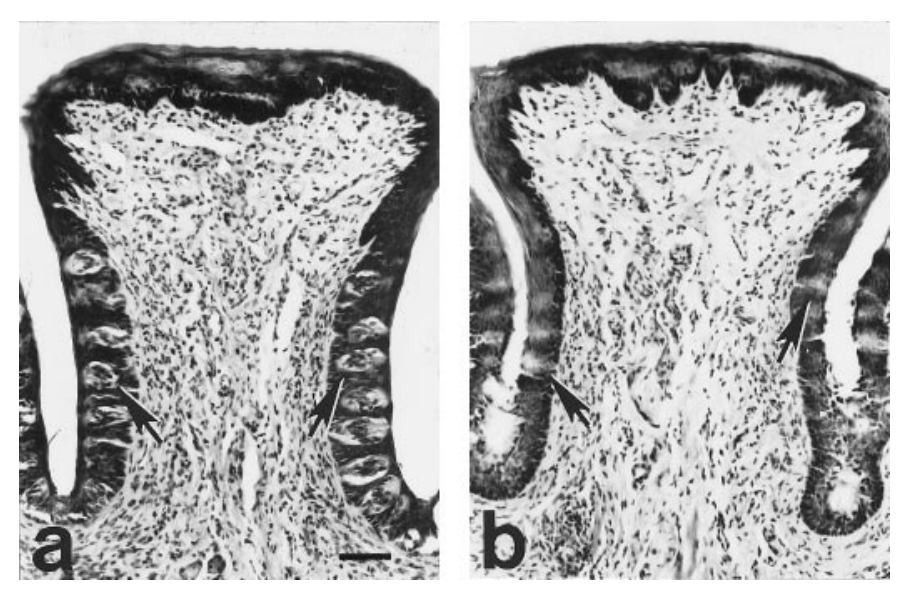

Figure 2. Photomicrographs of transverse sections through the circumvallate papilla stained with hematoxlyin and eosin from a sham-operated rat $(a)$ and a GL-transected rat $(b)$. There were numerous taste buds (arrows point to several) containing taste cells in the circumvallate papilla of control rats $(a)$. In $b$, the efficacy of GL transection is illustrated: no taste buds were apparent in the circumvallate papilla of GLX or DBLX rats. Frequently, "ghost" buds, unstained silhouettes of previously existing taste buds, were observed (arrows point to several of them). Scale bar, $125 \mu \mathrm{m}$.

the present study) distilled water (SHAM-W; Fig. 4, bottom set of dashed lines) evoked a significant increase in FLI neurons compared with nonstimulated rats $(p<0.02)$, providing definitive evidence that the somatosensory aspects of fluid stimulation contribute to Fos expression in the gNST. This increase is negligible, however, compared with the copious expression of Fos elicited by quinine stimulation, which was more than 2 times greater than that observed in distilled water-stimulated rats $(p<0.03)$. Thus, the chemical nature of the quinine solution was critical for enhanced FLI in the gNST.

Examination of individual rostrocaudal levels of the gNST demonstrated that quinine stimulation elevated Fos expression more so than did either no-stimulation or distilled waterstimulation $(p<0.02$ and $p<0.04$, respectively for each level, Fig. $5 a-d$ ). Only within the NgNST did quinine and distilled water evoke similar numbers of FLI neurons (Figs. 5e, 6). These data lend support for the supposition that enhanced FLI in the gNST was a taste-related phenomenon. Although quantitative analyses were not performed, it is noteworthy that progressively greater numbers of FLI neurons were found as more caudal sections within gNST were examined (Fig. $5 a-d$ ). This difference, however, likely reflects the increase in the size of the NST caudally.

Medial portions of the gNST showed significantly more FLI neurons than lateral regions (Fig. 7). Specifically, quinine was no more effective than distilled water in eliciting FLI in subfields 1 , 2 , and 3 (Fig. $7 a-c$ ). On the contrary, in subfields 4,5 , and 6 , a very different picture emerged. A robust and numerous population of FLI neurons was observed. Significantly more FLI neurons were observed in quinine-stimulated rats (SHAM-Q) compared with both nonstimulated (SHAM-NS; $p<0.02$ ) and distilled water-stimulated (SHAM-W; $p<0.03$ ) rats within each of these subfields, especially within subfield 5, which contained the greatest number of FLI neurons (Fig. $7 d-f$ ). A similar pattern of results was generally found throughout the rostrocaudal extent of each of these subfields (Fig. $8 a-l$ ) with several qualifications. In subfield 4 (Fig. $8 a-d$ ), the difference between SHAM-Q and SHAM-W rats at $\mathrm{RgNST}$ was not significant, and at IRgNST and CgNST the 

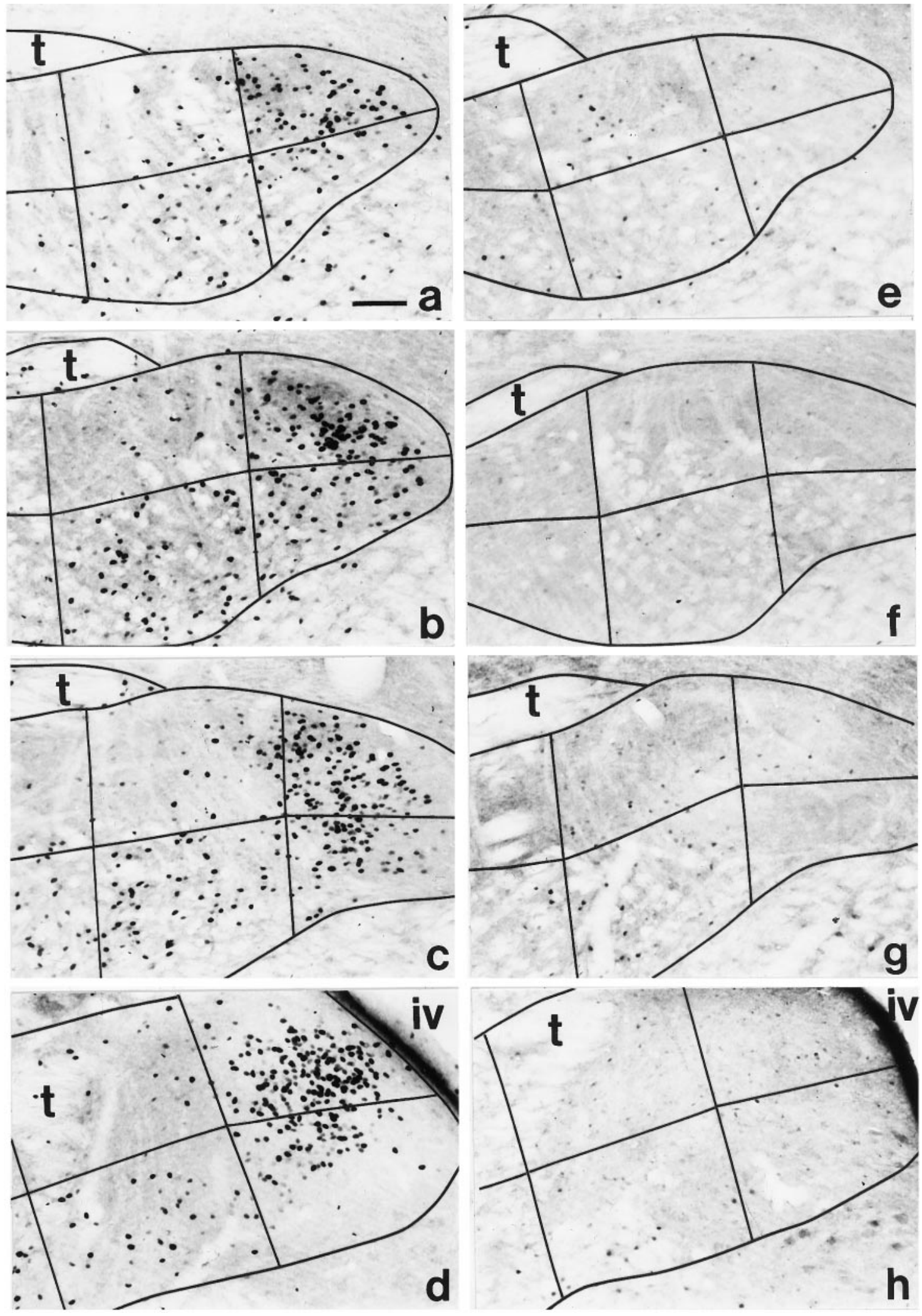

Figure 3. Photomicrographs of FLI in coronal sections through each level of the gNST examined in a quinine- and distilled water-stimulated rat. $a, e$, Rostral gNST; $b, f$, intermediate rostral gNST; $c, g$, intermediate caudal gNST; and $d, h$, caudal gNST. In $a-d$, robust FLI is demonstrated in this rat stimulated with $0.003 \mathrm{M}$ quinine. There was predominantly a dorsomedial (subfield 5) and midventral (subfield 4) distribution of the quinine-evoked FLI within the gNST (refer to Fig. $1 a$ for numbering of subfields). In contrast, very few FLI neurons were found in this distilled water-inf used rat ( $e-h$ ). Note that portions of the gNST, especially the lateral (left) and ventral borders (down), are outside the area pictured. $i v$, Fourth ventricle; $t$, solitary tract. Scale bar, $100 \mu \mathrm{m}$. 
Figure 4. Summed mean total $( \pm \mathrm{SE})$ number of FLI neurons observed across the four standardized rostrocaudal levels of the gNST. Top set of three dashed lines represents the mean ( $\pm \mathrm{SE}$ ) for SHAM-Q rats, and the bottom set depicts the mean \pm SE for SHAM-W rats. There was a main effect of treatment (using all seven groups) on the number of FLI neurons $\left(F_{(6,33)}=5.72\right.$; $p<0.0004)$. Asterisks indicate a significant difference $(p<0.05)$ from SHAM-Q value; pound sign denotes statistical differences $(p<0.05)$ compared with SHAM-W values.

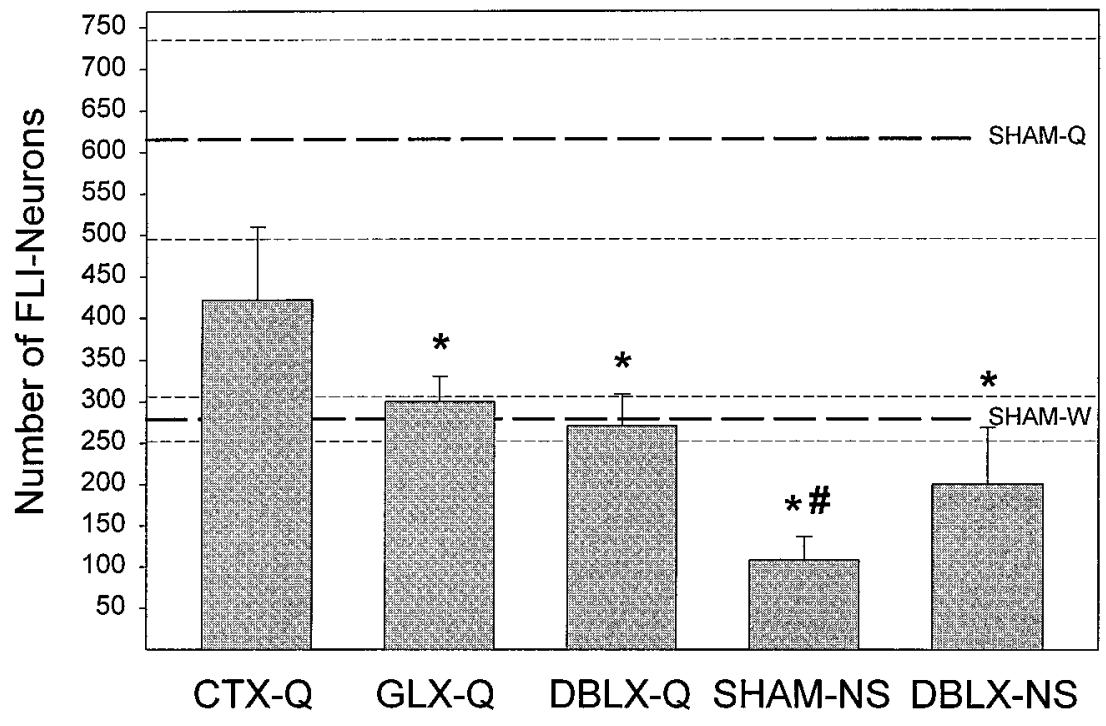

difference just missed the statistical rejection criteria (both, $p=$ 0.07). Similarly in RgNST of subfield 6 (Fig. $8 i$ ), the number of FLI neurons in SHAM-Q and SHAM-W rats was not significantly different.

\section{Distribution of FLI neurons}

FLI neurons, regardless of their number, were distributed in distinctive patterns within the gNST depending on the tastant delivered (Figs. 3, 9). That is, when the data from each subfield were plotted as a proportion of the total number of FLI neurons in the gNST, a "fingerprint" of FLI expression was revealed for each stimulus condition (Fig. $9 a-c$ ). As mentioned previously, quinine-evoked FLI neurons (SHAM-Q) were concentrated within the medial portions of the gNST (subfields 4, 5, and 6) and distributed as an oblique band of cells starting in the dorsomedial portion of the nucleus (subfield 5) and extending ventrolaterally through subfield 4 into the reticular formation (Fig. $3 a-d$ ). While subfields 4 and 5 contained the greatest proportion of FLI neurons in quinine-stimulated rats (Fig. 9a), the pattern of FLI expression differed considerably in the other SHAM groups. In distilled water-stimulated rats (SHAM-W; Fig. 9b), subfields 2 and 4 contained the greatest proportion of FLI neurons, whereas in nonstimulated rats (SHAM-NS; Fig. 9c), the majority of FLI neurons were found in subfields 3 and 4.

\section{Staining intensity of FLI neurons}

As a complement to counting and observing the distribution of FLI neurons, the effects of the various conditions were also assessed by quantifying the staining intensity of the label. SHAM-Q rats showed a greater proportion of dark staining FLI neurons $(23 \%)$ compared with both SHAM-W $(10 \% ; p<0.01)$ and SHAM-NS (5\%; $p<0.0001)$ rats. Moreover, SHAM-Q rats showed a significantly smaller proportion of light staining FLI neurons (20\%) within the gNST as compared with both SHAM-W (45\%; $p<0.001)$ and SHAM-NS $(47 \% ; p<0.001)$ rats. There were no differences in the proportion of neurons classified as intermediate in staining intensity.

\section{FLI in subdivisions versus subfields}

Notably, the distribution of quinine-evoked FLI neurons in the gNST in the present study was in accord with that reported by Harrer and Travers (1996). These authors reported elevated FLI within medial portions of the gNST, specifically in the medial half of RC. The RC subdivision of the NST receives dense projections from gustatory afferent fibers in the hamster (Whitehead, 1988). When the spatial distribution of FLI neurons in the gNST for two quinine-stimulated rats was analyzed using these same anatomically defined subdivisions, the proportion of quinine-evoked FLI neurons in each subdivision was remarkably similar to that reported by Harrer and Travers (1996). In both studies, more than half of all quinine-evoked FLI neurons were found in RC, whereas about one-third were observed in $\mathrm{V}$ (Table 3 ). It is noteworthy that portions of subfields 4,5 , and 6 collectively captured $>76 \%$ of the quinine-evoked cells distributed in RC, and the majority of these were found in subfield 5 (Table 3, Figs. 1,3). The proportions of distilled water-stimulated FLI neurons and nonstimulated FLI neurons in the four subdivisions of the gNST in the present study were distinct from each other and from the proportions of quinine-stimulated neurons as demonstrated by Harrer and Travers (1996) (Table 3).

\section{The effects of nerve transection on FLI neurons in the NST}

\section{Number of FLI neurons}

It is important to note first that nerve transections, in the absence of any oral stimulation, do not appear to influence the expression of FLI in the gNST, as evidenced by the results of the DBLX-NS rats. That is, combined GL and CT neurotomy (DBLX-NS) did not significantly affect the total number of FLI neurons in the gNST compared with SHAM-NS rats ( $p>0.5$; Fig. 4). However, when quinine was used as a tastant, gustatory nerve transections had dramatic effects on FLI neurons (Fig. 10). In a preliminary report, we noted that CTX, GLX, and DBLX were equally effective in attenuating the mean total number of FLI neurons in the gNST after intraoral infusion of quinine (King et al., 1997); however, with an increase in sample size, the use of more conservative statistical tests and more detailed analyses of the data (i.e., parcellation of the gNST into subfields), it became clear that although bilateral transection of the $\mathrm{CT}$ reduced the number of FLI neurons in the gNST to a level close to that after distilled water infusion, the effect was modest relative to that resulting from bilateral GLX or DBLX (Fig. 10). As shown in Figure 4, GLX and DBLX dramatically decreased the mean total of 


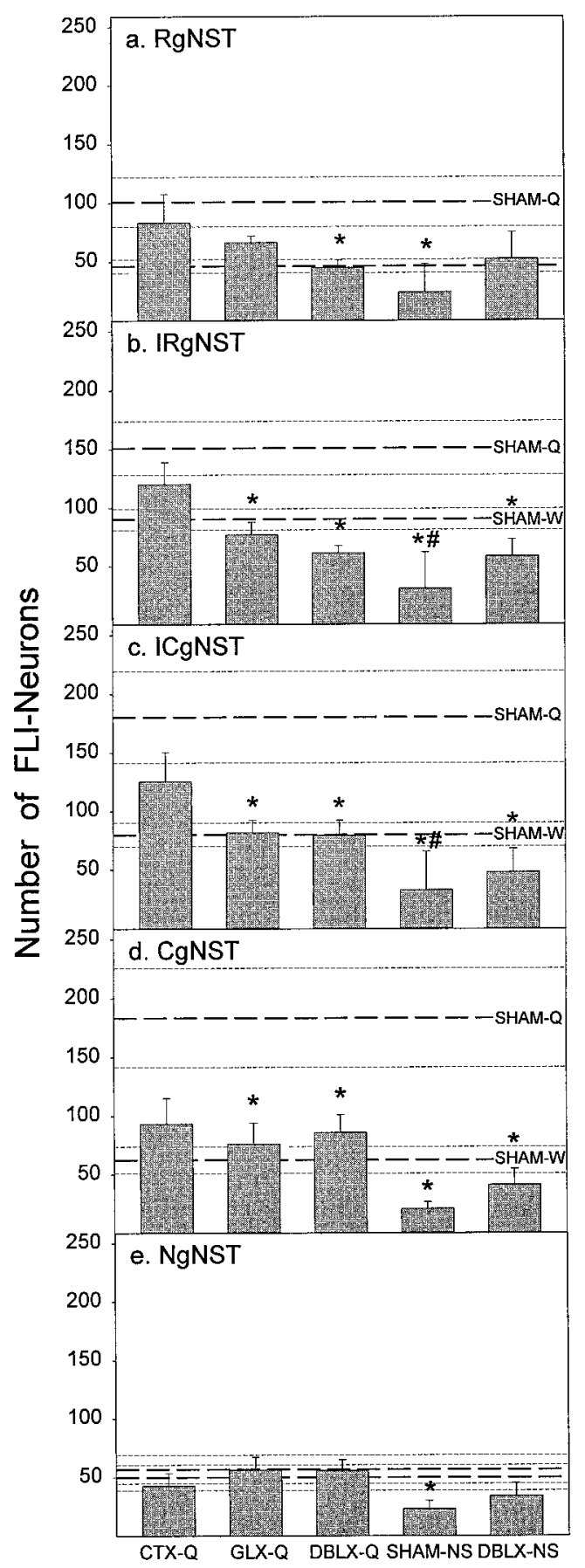

Figure 5. Mean ( \pm SE) number of FLI neurons observed at each of the standard rostrocaudal levels of the gNST. In each panel, the top set of three dashed lines represents the mean ( \pm SE) for SHAM-Q rats, and the bottom set depicts the mean \pm SE for SHAM-W rats. The following ANOVA values represent the main effect of treatment on the number of FLI neurons for each rostrocaudal level; all seven groups were included. Asterisks indicate a significant difference $(p<0.05)$ from SHAM-Q value; pound signs denote statistical differences $(p<0.05)$ compared with SHAM-W values. $a$, RgNST $\left(F_{(6,33)}=2.71 ; p<0.03\right) ; b$, IRgNST $\left(F_{(6.33)}=7.79 ; p<0.00003\right) ; c$, ICgNST $\left(F_{(6,33)}=5.39 ; p<0.0006\right) ; d$, CgNST $\left(F_{(6,33)}=5.49 ; p<0.0005\right)$. Note that across each level quinine (SHAM-Q) elicited significantly more FLI neurons compared with both distilled-water (SHAM-W) and nonstimulated rats (SHAM-NS). Only within the nongustatory NST (NgNST) did quinine not elicit significantly more FLI neurons than distilled water inf usion $(p>0.67)$. Additionally, no main effect of treatment was found $\left(F_{(6,33)}=1.39 ; p>0.25\right)$ at this level. quinine-elicited FLI neurons in the gNST compared with SHAM-Q animals ( $p<0.03$ and $p<0.02$, respectively). Interestingly, the number of FLI neurons remaining after GLX or DBLX was nearly identical to the number of FLI neurons observed in distilled water-stimulated rats (SHAM-W). On the contrary, CTX did not significantly reduce the number of FLI neurons elicited by quinine infusion relative to sham-operated controls, although it did reduce it to a number that was not different from that after distilled water infusion. These results indicate that, overall, CT transection had an intermediate effect on the expression of quinine-evoked FLI in the gNST.

This same pattern of nerve transection effects was apparent at intermediate levels of the gNST examined (Fig. 5b,c). That is, in IRgNST and ICgNST, both GLX and DBLX significantly attenuated FLI neurons in quinine-stimulated rats to a number close to that found in distilled water-infused rats $(p<0.02$, for each comparison), whereas FLI in CTX rats was not different from SHAM rats stimulated with either water (SHAM-W) or quinine (SHAM-Q). In RgNST (Fig. 5a), neither CT nor GL transection alone produced a statistically significant decrease in FLI neurons compared with the SHAM-Q group; however, neither group was significantly different compared with distilled water-infused rats either, indicating an intermediate effect of the individual nerve transections on FLI in RgNST. Only combined neurotomy produced a significant attenuation of the number of FLI neurons at this most rostral level of the gNST $(p<0.03)$. Within CgNST (Fig. 5d), GLX and DBLX resulted in similar decreases of FLI $(p<0.05)$. Surprisingly, CT transection also produced a near significant effect $(p=0.08)$ at this caudal level. No gustatory nerve transection affected FLI in NgNST (Fig. 5e).

Because quinine and distilled water were equally effective in eliciting FLI in subfields 1, 2, and 3, it is not surprising that gustatory nerve transections did not affect FLI in these lateral subregions (Fig. $7 a-c$ ). In subfields 4,5 , and 6 , where the majority of quinine-evoked FLI neurons were found, gustatory nerve transection had interesting effects (Fig. $7 d-f$ ). In subfields 4 and 6 , GLX resulted in significant reductions in FLI after intraoral infusions of quinine (both, $p<0.03$ ). The addition of CT transection did not further attenuate the number of quinine-evoked FLI neurons found in GL-transected rats. As reported for Total gNST (Fig. 4), CTX alone produced results intermediate between SHAM-Q and SHAM-W rats. This pattern of nerve transection effects was maintained across all rostrocaudal levels of these two subfields with a few notable exceptions (Fig. 8). In subfield 4, no nerve transection was effective in significantly reducing FLI in RgNST (Fig. 8a), while in CgNST, CTX was as effective as GLX in attenuating FLI $(p<0.04$; Fig. $8 d)$. In subfield 6, only DBLX was effective in attenuating FLI neurons in $\operatorname{RgNST}(p<0.04$; Fig. 8i).

Because a large proportion of FLI neurons were observed in subfield 5, it was anticipated that nerve transections might have their greatest effect within this subfield. The number of FLI neurons in subfield 5 after intraoral infusions of quinine was severely attenuated by GLX $(p<0.01$; Fig. $7 e)$. Nearly all of the quinine-evoked FLI across the rostrocaudal extent of this subfield in the gNST was eliminated (Fig. $8 e-h$; RgNST, $p<0.02$; IRgNST, $p<0.001$; ICgNST, $p<0.003$; CgNST, $p=0.059$ ). Once again, the addition of $\mathrm{CT}$ transection to GL transection did not result in a greater reduction of FLI compared with GLX rats. Transection of the CT alone had little, if any, effect on the number of FLI neurons within subfield 5. Once again, the number of FLI neurons in SHAM-Q rats was not significantly different 

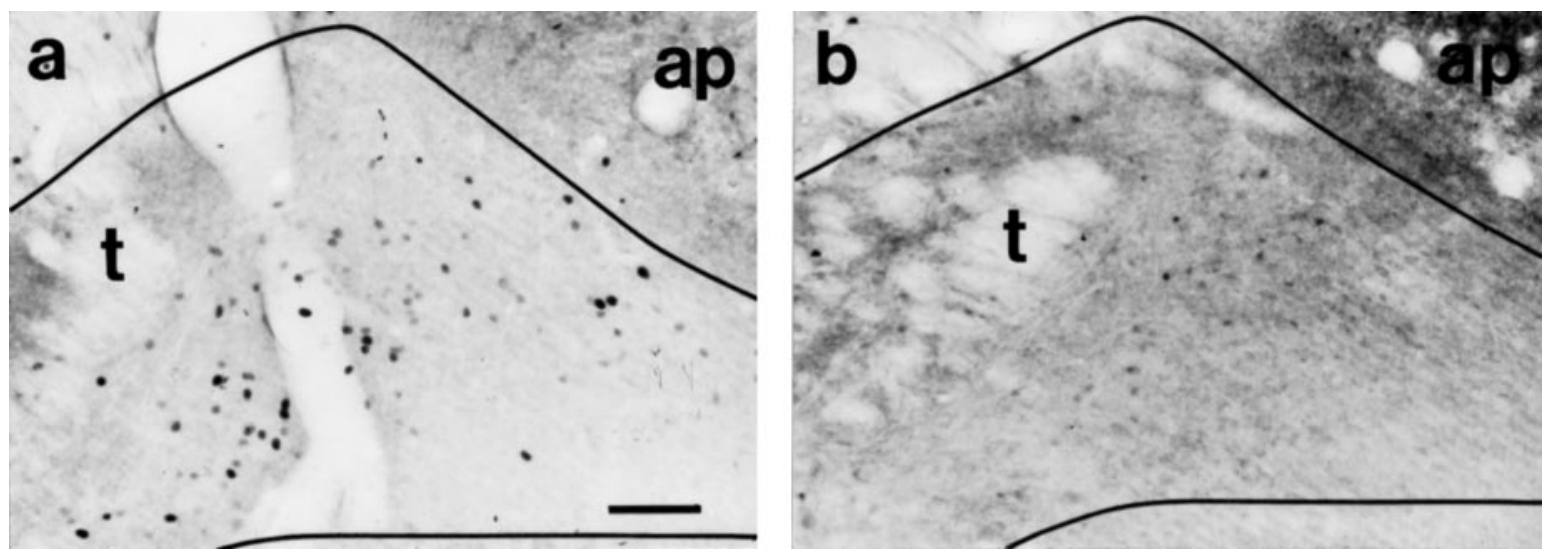

Figure 6. Photomicrographs of FLI in coronal sections through the nongustatory level of the NST examined in the same quinine-stimulated ( $a$ ) and distilled water-stimulated $(b)$ rats as in Figure 3. Because so few FLI neurons were apparent at this level after stimulation with either tastant, no subfield analyses were performed. Note that portions of the nucleus, especially the lateral border (left), are outside the area pictured. $a p$, Area postrema; $t$, solitary tract. Scale bar, $100 \mu \mathrm{m}$.

from that found in CTX-Q rats. However, the number of quinineelicited FLI neurons in CTX-Q rats was significantly greater compared with SHAM-W rats $(p<0.02$; Fig. $7 e)$, specifically within the intermediate portions of gNST $(p<0.001$, IRgNST; $p<0.01$, ICgNST; Fig. $8 f g$ ). These results indicate that in subfield 5, CTX does not produce an intermediate effect. Rather, CTX had very little effect on the number of quinine-elicited FLI neurons in subfield 5 (Fig. 7e), especially at intermediate levels of gNST (Fig. 8f,g).

\section{Distribution of FLI neurons}

Interestingly, the relative spatial distribution of FLI across the subfields of the gNST, irrespective of the number of FLI neurons, differed in distinct ways among the groups (Figs. 9, 10). In both GLX-Q (Fig. 9e) and DBLX-Q (Fig. 9f) rats, a dramatic shift from the SHAM-Q (Fig. 9a) to the SHAM-W (Fig. 9b) pattern was observed. In striking contrast, there was remarkable concordance between the profiles of SHAM-Q and CTX-Q rats (Fig. 9a,d).

\section{Staining intensity of FLI neurons}

Compared with SHAM-Q rats that had $\sim 23 \%$ of their FLI cells classified as dark staining, the proportion of dark staining FLI neurons in CTX-Q (8\%), GLX-Q (7\%) and DBLX-Q (4\%) was significantly smaller (all, $p<0.002$ ). Moreover, both GLX and DBLX rats had $\sim 44 \%$ of their FLI neurons classified as light staining. This represents significant increases in the proportion of light staining quinine-evoked FLI neurons compared with SHAM-Q rats (both, $p<0.004$ ), which had only $21 \%$ of their FLI neurons staining lightly. The proportion of light staining FLI neurons in CTX-Q rats (35\%), however, was not significantly different from that found in SHAM-Q rats. Thus, in this measure as was the case in the counts, CTX appeared to have an intermediate effect on FLI relative to GLX or DBLX. The proportion of intermediate staining FLI neurons was not different among the groups.

\section{DISCUSSION}

\section{FLI in gNST is taste-related}

The data from the present experiment provide convincing evidence that FLI in the gNST after intraoral infusion of quinine is related primarily to oral stimulation rather than postingestive events. Notably, throughout the rostrocaudal extent of the gNST, FLI was enhanced after quinine infusion compared not only with nonstimulated animals, but importantly, compared with distilled water-infused animals as well. Furthermore, quinine evoked the same degree of FLI as distilled water only within the nongustatory NST. Although the somatosensory aspects of fluid stimulation did contribute to the elevated FLI in the gNST, the chemical, presumably gustatory, properties of quinine were more consequential. Harrer and Travers (1996) came to a similar conclusion based on their results, which showed progressive increases in the number of FLI neurons in the unstimulated, water-, and quinineor sucrose-stimulated groups, although only the differences between the unstimulated and taste-stimulated groups were statistically significant. Perhaps the most compelling evidence for the supposition that FLI in the gNST is related to "taste" was the finding that gustatory nerve transections had profound consequences. If postoral events had been the principal stimuli eliciting FLI, then gustatory nerve transections would have had little, if any, effect. On the contrary, bilateral transection of the GL alone, for example, essentially eliminated FLI neurons in the gNST, dramatically altered their distribution, and reduced the proportion of dark staining FLI neurons in the nucleus. Collectively, these results confirm and extend the findings of Harrer and Travers (1996) by demonstrating that the increase in FLI in the gNST after intraoral quinine infusions has a gustatory basis.

Certainly, these interpretations, as well as those that follow, must be tempered by the limitations of the Fos technique. Of primary concern is that stimulus-induced $c$-fos expression occurs only in those neurons that generate a sufficient amount of neuronal activity. Accordingly, very high stimulus intensities must typically be used to achieve measurable FLI. Moreover, in some brain regions, neurons remain Fos-immunonegative regardless of the strength or duration of the stimulus (Dragunow and Faull, 1989). The reasons for this are unclear, but may be related to biochemical messengers that regulate $c$-fos activation in neurons (Dragunow and Faull, 1989) or to the preferential expression of transcription factors other than c-fos (Sheng and Greenberg, 1990; Morgan and Curran, 1991; Kaczmarek and Chaudhuri, 1997). Thus, in the current investigation, it is possible that cells were neurally activated by quinine infusion but escaped detec- 


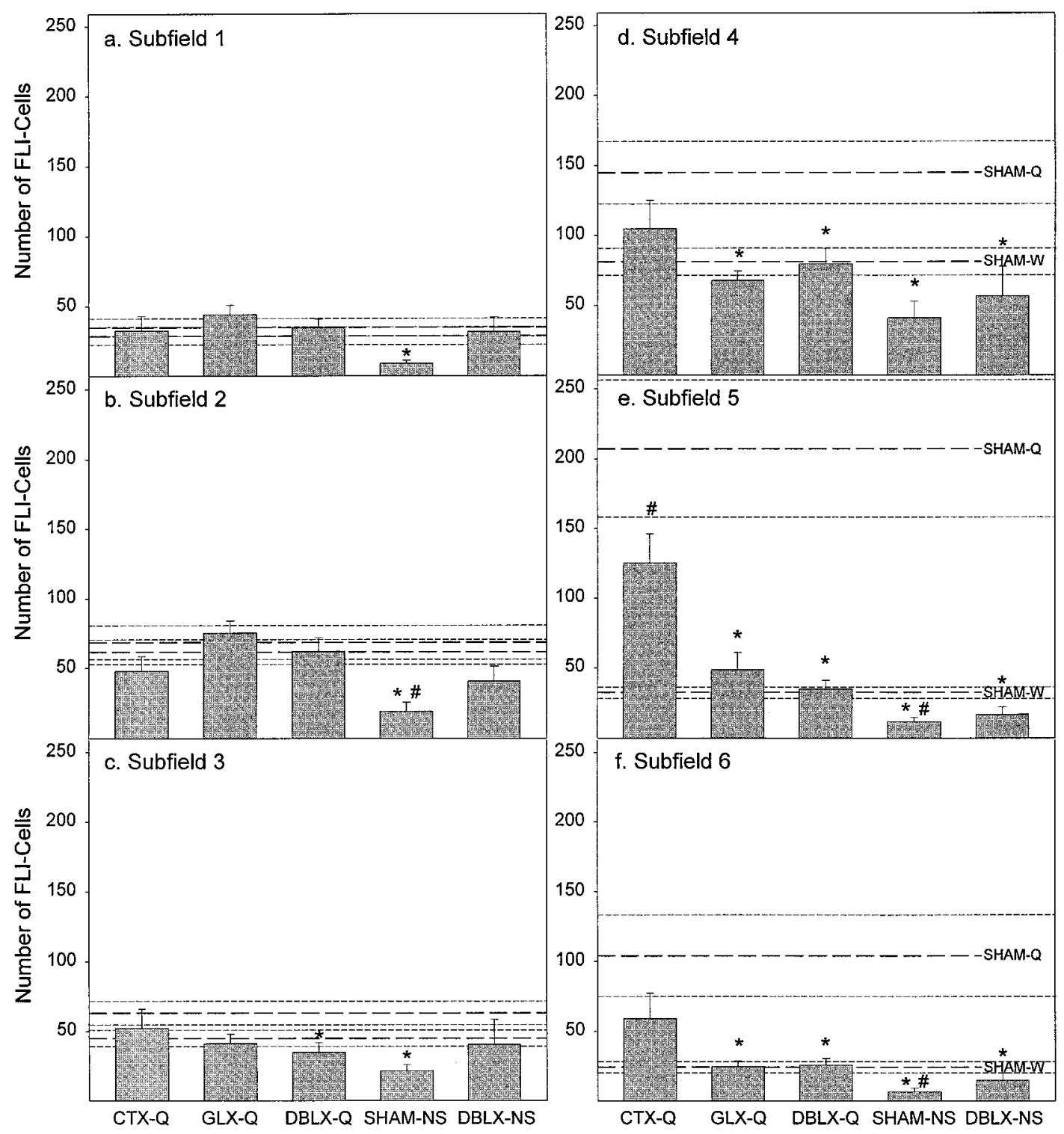

Figure 7. Summed mean $( \pm \mathrm{SE})$ total number of FLI neurons observed for each subfield pooled across the four standardized rostrocaudal levels. In each panel the top set of three dashed lines represents the mean ( \pm SE) for SHAM-Q rats, and the bottom set depicts the mean $( \pm$ SE) for SHAM-W rats. The following ANOVA values represent the main effect of treatment on the number of FLI neurons for each subfield examined; all seven groups were included. Asterisks indicate a significant difference $(p<0.05)$ from SHAM-Q value; pound signs denote statistical differences $(p<0.05)$ compared with SHAM-W values. In subfield $1(a)\left(F_{(6,33)}=1.92 ; p>0.10\right)$, subfield $2(b)\left(F_{(6.33)}=3.51 ; p<0.008\right)$, and subfield $3(c)\left(F_{(6,33)}=1.68 ; p>0.15\right)$ quinine (SHAM-Q) did not elicit more FLI neurons than distilled water (SHAM-W). In contrast, in subfield $4(d)\left(F_{(6,33)}=4.67 ; p<0.002\right)$, subfield $5(e)\left(F_{(6,33)}=10.74 ; p<0.000001\right)$, and subfield $6(f)\left(F_{(6,33)}=5.81 ; p<0.0004\right)$ quinine inf usion (SHAM-Q) resulted in significantly more FLI neurons compared with either distilled water (SHAM-W) or no stimulation (SHAM-NS). Note the overlap in the number of FLI neurons between SHAM-Q and SHAM-W rats in subfields 1,2 , and 3 and the impressive difference between them in subfields 4, 5, and 6 .

tion. Nevertheless, within these limitations, the results are not without meaning. Although the Fos-positive neurons may only represent a subpopulation of all the neurons activated by quinine, it is still informative to ask how this subpopulation is spatially distributed within the gNST under a fixed stimulus condition and to assess the consequences of specific nerve transection on that distribution. Accordingly, this research strategy led to some interesting topographical relations between peripheral gustatory input and central patterns of neuronal activation in the gNST.

\section{Quinine-elicited FLI in gNST: topographic organization}

If the induction of immediate early genes such as c-fos is strongly, although not exclusively linked to neuronal activation, it follows that transection of the GL would dramatically reduce the number of Fos-positive cells after infusion of quinine because this nerve is the most electrophysiologically responsive of the gustatory nerves to this tastant. Moreover, one might predict that enhanced FLI would be found primarily within the intermediate portions of the rostral NST because the GL has its central terminations here (Contreras et al., 1982, Hamilton and Norgren, 1984). As it turned out, the quinine-evoked Fos response was not only attenuated, but was virtually eliminated by GL transection not only within the intermediate gNST but across all levels of the gNST examined (Figs. 5, 7, 8, 10). Furthermore, the distribution of the few FLI neurons remaining after GL transection was strikingly similar to the distribution of FLI neurons in distilled water- 


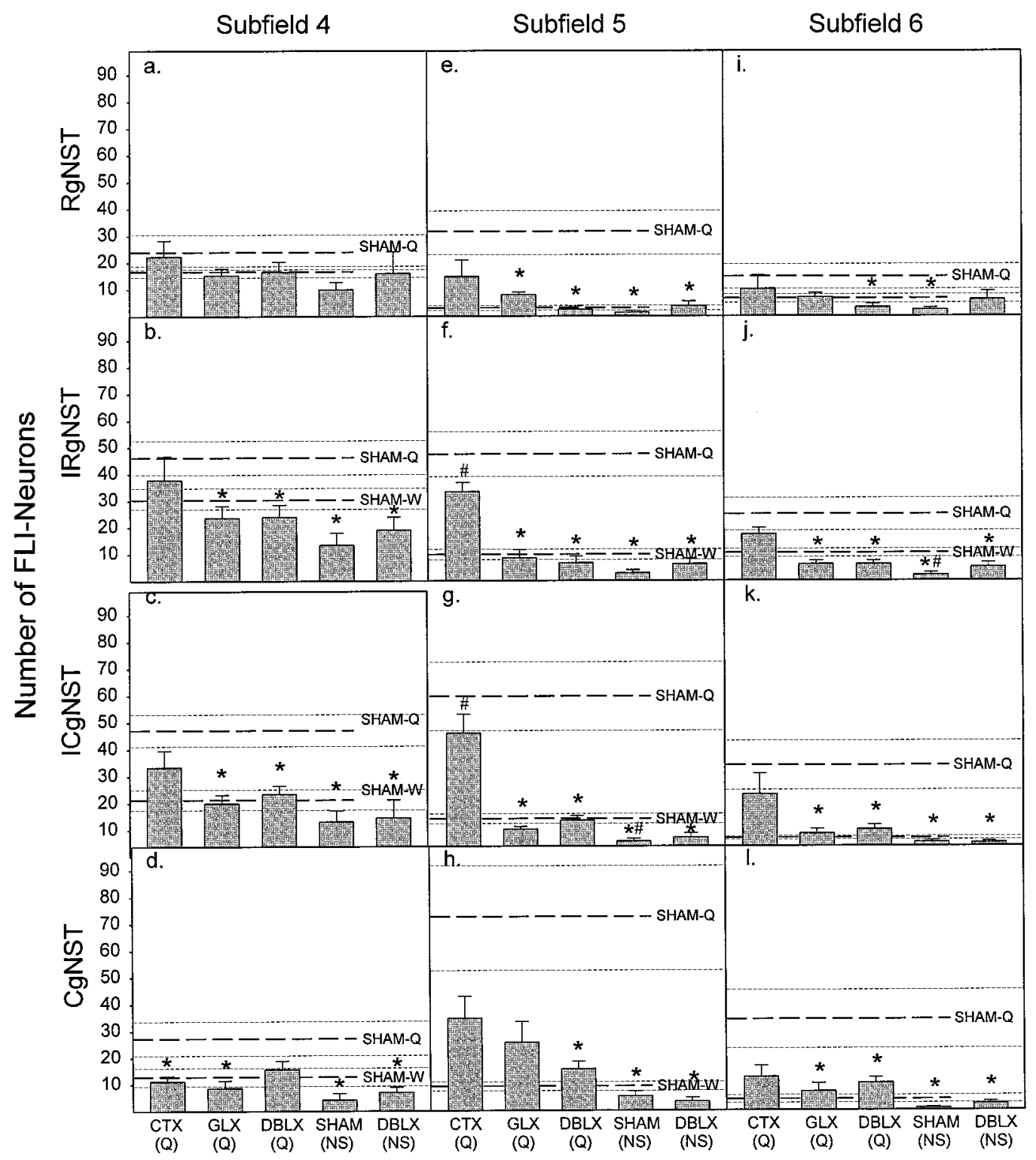

Figure 8. Mean ( \pm SE) number of FLI neurons observed at each rostrocaudal level of the gNST for subfield $4(a-d)$; subfield $5(e-h)$, and subfield 6 $(i-l)$. In each panel the top set of three dashed lines represents the mean $( \pm$ SE) for SHAM-Q rats, and the bottom set depicts the mean $( \pm$ SE) for SHAM-W rats. The following ANOVA values represent the main effect of treatment on the number of FLI neurons for each subfield at each rostrocaudal level examined; all seven groups were included. Asterisks indicate a significant difference $(p<0.05)$ from SHAM-Q value; pound signs denote statistical differences $(p<0.05)$ compared with SHAM-W values. $a$, RgNST-4 $\left(F_{(6.33)}=0.87 ; p>0.52\right) ; b, \operatorname{IRgNST}-4\left(F_{(6.33)}=3.80 ; p<0.006\right) ; c$, ICgNST-4 $\left(F_{(6,33)}=6.02 ; p<0.0003\right) ; d$, CgNST-4 $\left(F_{(6,33)}=4.41 ; p<0.003\right) ; e$, RgNST-5 $\left(F_{(6,33)}=6.05 ; p<0.0002\right) ; f$, IRgNST $-5\left(F_{(6,33)}=17.45 ; p<0.0000001\right) ;$ $g$, ICgNST-5 $\left(F_{(6,33)}=13.19 ; p<0.0000001\right) ; h, \operatorname{CgNST}-5\left(F_{(6,33)}=6.58 ; p<0.0002\right) ; i, \operatorname{RgNST}-6\left(F_{(6,33)}=1.82 ; p>0.13\right) ; j$, IRgNST-6 $\left(F_{(6,33)}=8.10 ;\right.$ $p<0.00002) ; k$, ICgNST-6 $\left(F_{(6,33)}=5.23 ; p<0.0008\right) ; l$, CgNST-6 $\left(F_{(6,33)}=5.40 ; p<0.0006\right)$.

infused rats (Fig. 9), suggesting that the "neural representation" of quinine was indistinct from water in this subpopulation of cells after GL transection. This latter interpretation, of course, leads to behavioral predictions that are discussed below. In addition, these results imply that the GL provides input to all cells expressing FLI in response to the concentration of quinine used in this study.

Because the CT does not respond robustly to quinine, it was not surprising to find that its transection did not produce as great a reduction in the number of quinine-evoked FLI neurons as GL transection (Figs. 4, 5, 7, 8, 10). In fact, CT transection essentially had no effect on the number of quinine-evoked FLI neurons within subfield 5 (where quinine stimulation elicited the greatest
Fos response). Nonetheless, this nerve must provide input to at least some of the quinine-evoked FLI neurons because its transection did decrease FLI at each rostrocaudal level of the gNST examined, albeit not as unequivocally as GL transection (Figs. 5, $7,8,10)$. It is particularly noteworthy that although CT transection attenuated the number of quinine-elicited FLI neurons, their spatial distribution did not deviate from the distinctive quinineassociated pattern found in sham-operated rats (Fig. 9), unlike the consequences of GL transection. This suggests that the neural representation of quinine after CT transection is still comparable to that in sham-operated controls and remains distinguishable from the neural representation of water. 


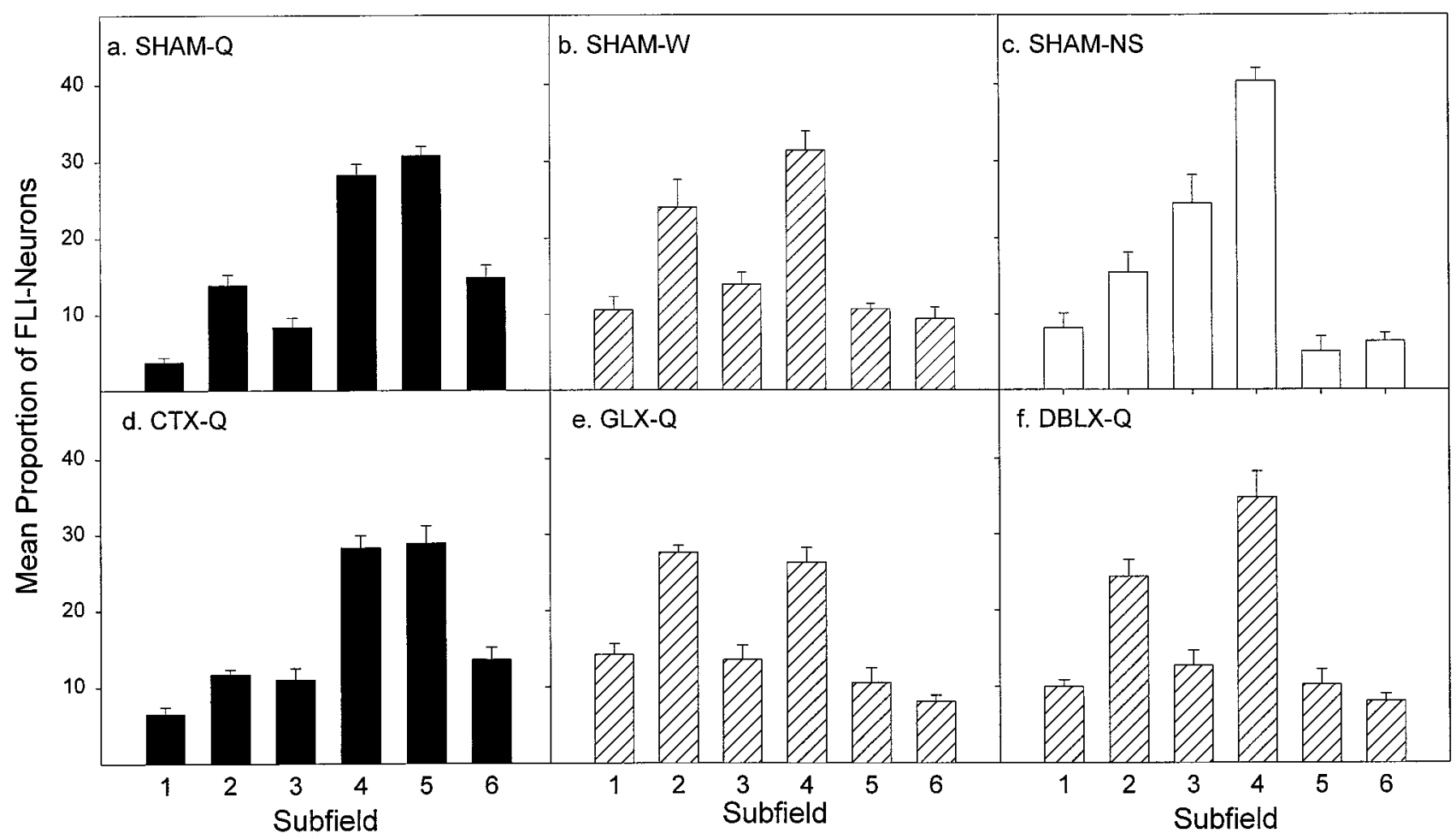

Figure 9. Mean $( \pm$ SE) proportion of FLI neurons in each subfield for each stimulus condition. Proportions were calculated by dividing the number of FLI neurons observed in each subfield by the total number of FLI neurons in the gNST for each animal and then averaged for each stimulus condition. SHAM-Q $(a)$, SHAM-W $(b)$, and SHAM-NS $(c)$ rats elicited distinct patterns of FLI, depending on the condition. Notably, the distribution of FLI in CTX-Q rats $(d)$ faithfully reflected that found in SHAM-Q rats. This pattern is denoted with black bars. Although few in number, FLI neurons in GLX-Q $(e)$ and DBLX-Q $(f)$ rats were distributed similarly, and their FLI pattern mirrored that found in SHAM-W rats $(b)$. This pattern is denoted with cross-hatched bars.

Table 3. Mean proportion of FLI neurons in gNST subdivisions

\begin{tabular}{llll} 
& \multicolumn{3}{l}{ Proportion of FLI Neurons in gNST $^{a}$} \\
\cline { 2 - 4 } & $\begin{array}{l}\text { SHAM-Q } \\
(n=2)\end{array}$ & $\begin{array}{l}\text { SHAM-W } \\
(n=2)\end{array}$ & $\begin{array}{l}\text { SHAM-NS } \\
(n=1)\end{array}$ \\
\hline Medial & $0.09(0.05)^{b}$ & $0.05(0.07)^{b}$ & $0.00(0.04)^{b}$ \\
RC & $0.51(0.55)^{b}$ & $0.36(0.44)^{b}$ & $0.31(0.23)^{b}$ \\
Ventral & $0.32(0.31)^{b}$ & $0.38(0.35)^{b}$ & $0.52(0.55)^{b}$ \\
RL & $0.09(0.09)^{b}$ & $0.22(0.14)^{b}$ & $0.18(0.18)^{b}$
\end{tabular}

$\overline{{ }^{a} \text { Means were calculated using only the three most rostral sections of the gNST }}$ analyzed in the present study. These sections roughly correspond to those analyzed in the Harrer and Travers (1996) study.

${ }^{b}$ Based on data reported by Harrer and Travers (1996).

The finding that the number of FLI neurons was attenuated to some degree after either GL or CT transection but that combined neurotomy did not reduce their number more than single nerve transections alone supports the existence of some degree of convergence between the inputs of these two nerves. In theory, it is possible that all quinine-related Fos-positive NST neurons receive input from both nerves, but the contribution of the $\mathrm{CT}$ is relatively weaker, such that removal of its activity does not affect the ability of some cells to display FLI. That is, although Fospositive neurons might receive input from the $\mathrm{CT}$ when it is intact, quinine-evoked activity from the GL may be sufficient to bring about Fos expression in some neurons when the $\mathrm{CT}$ is transected. Similarly, it is possible that some Fos-positive neurons receive input from either the GSP or the superior laryngeal nerve; if either of these nerves would have been transected alone or in combination with the CT, there may have been noticeable effects on the number, spatial distribution, or intensity of FLI neurons. Alternatively, neurons that display quinine-induced FLI after CT transection (for example, in subfield 5) may be exclusively influenced by GL activity. In either event, the results suggest that some neurons that display FLI after quinine stimulation receive input from both the CT and GL. Indeed, although extracellular recording studies provide only minimal evidence for convergence between gustatory signals arising from the VIIth and IXth nerves (Ogawa et al., 1984; Travers and Norgren, 1995; Travers et al., 1986), a recent study that used an in vitro patchclamp preparation to specifically target the VIIth/IXth overlap region found that most cells could be driven by electrical stimulation of both VIIth and IXth afferent fibers (Grabauskas and Bradley, 1996).

The proposed convergence of GL and CT input need not be the result of primary afferent fibers from these nerves impinging on the same second order neurons. Indeed, based on the anatomical positions of the central terminations of the gustatory nerves, in comparison with the distribution of quinine-induced Fos expression, this is unlikely. Although there is some overlap (Lasiter, 1992), the CT terminal field is confined largely to the rostral gNST and the GL terminal field is primarily located more caudally (Contreras et al., 1982; Hamilton and Norgren, 1984). It is interesting to note that within subfield 5 at the intermediate level of the gNST (where the GL principally terminates), CTX had no effect on quinine-elicited FLI while GLX did. Nevertheless, the chemotopic pattern of quinine-induced FLI does not strictly correspond with the orotopy defined by the terminal zones of the 

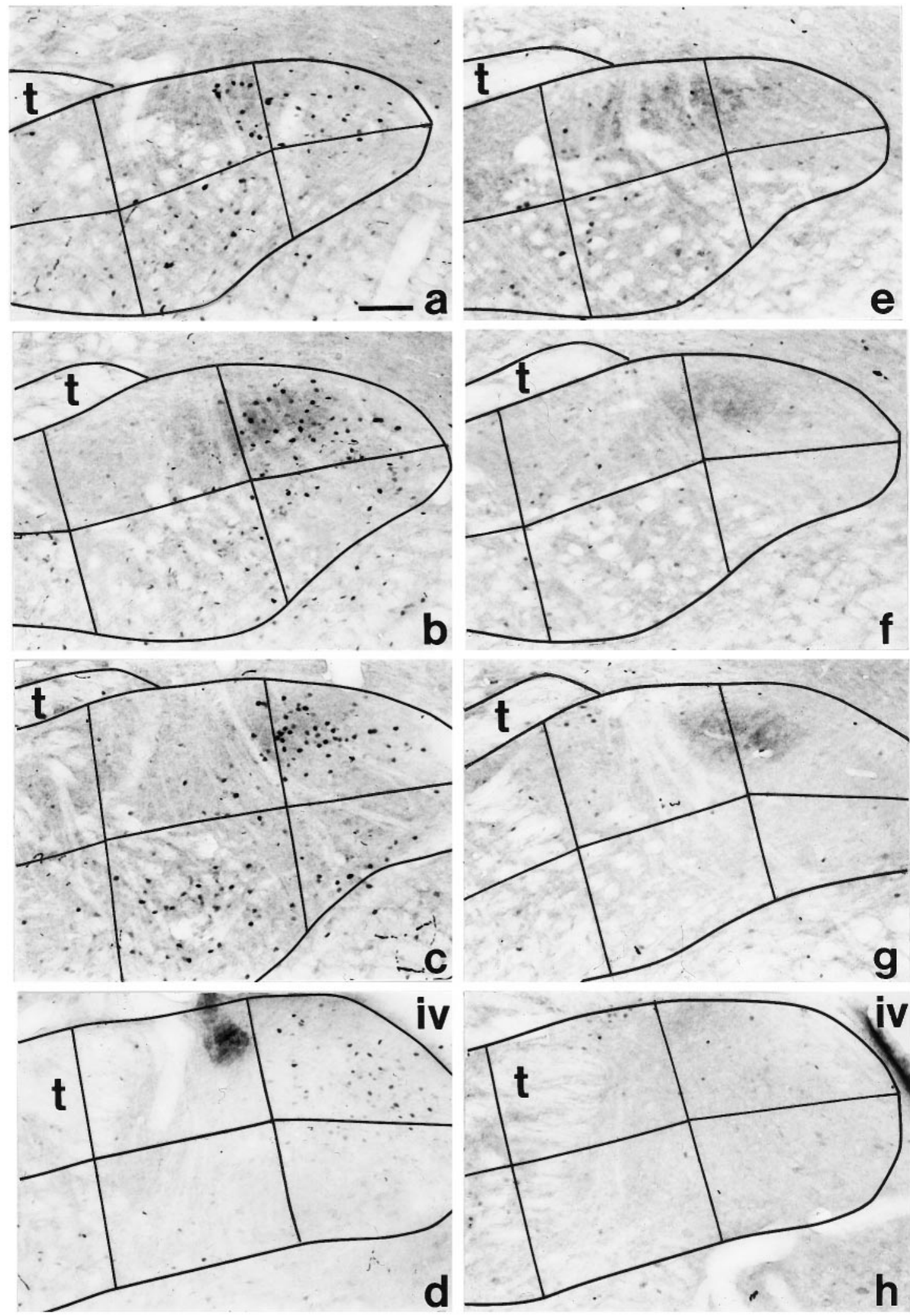

Figure 10. Photomicrographs of FLI in coronal sections from a quinine-stimulated CTX rat $(a-d)$ and a quinine-stimulated GLX rat $(e-h)$ across each rostrocaudal level of the gNST analyzed. $a, e$, Rostral gNST; $b, f$, intermediate rostral gNST; $c, g$, intermediate caudal gNST; and $d, h$, caudal gNST. CT transection did not eliminate, but did attenuate (see Fig. 3 for comparison) quinine-evoked FLI neurons in the gNST, and GL transection virtually eliminated them. FLI number and distribution in quinine-stimulated DBLX rats (data not shown) paralleled that found for GLX rats. Refer to Figure $1 a$ for numbering of subfields. Note that portions of the gNST border, especially the lateral (left) and ventral (down) borders are outside the area pictured. $i v$, Fourth ventricle; $t$, solitary tract. Scale bar, $100 \mu \mathrm{m}$. 
CT and GL. These findings are in agreement with Harrer and Travers (1996), who reported that the chemotopic patterns of FLI after quinine or sucrose stimulation were not simply reflections of the orotopic arrangement of primary afferent fibers within the gNST. That GL transection was effective in reducing FLI throughout the rostrocaudal extent of the gNST demonstrates that input from the GL can affect the responsiveness of NST neurons rostral to its primary terminal zone. Likewise, CT transection produced effects at the caudal level of the gNST, an area well removed from its primary afferent terminal field. Many FLI neurons were found in areas of the NST where only diffuse, if any, terminations from the GL or CT have been reported (e.g., ventrally, in subfield 4). Collectively, these anatomical realities weaken the notion that the proposed convergence occurs exclusively at the second order neurons.

The use of nerve transections to determine functional topography is not without its caveats. For example, peripheral nerve transection has been shown to lead to remarkable reorganizational events in the somatosensory system (Jain et al., 1998). Although there is evidence of anatomical consequences of gustatory nerve transection in the NST (Smith et al., 1995; Whitehead et al., 1995), it is unclear whether these changes can be considered reorganizational from a functional perspective. At issue is whether the pattern of activity, as defined by FLI, represents the normal pattern minus the input of the transected nerve or whether compensatory reorganizational events are influencing the pattern observed in the neurotomized animals. This question is difficult to answer at this time because the assessment of reorganizational events first requires a clear depiction of normal functional organization, and to date, this remains undefined. These issues are surely important when considering the interpretations of the results presented in this paper. Nevertheless, once a nerve is transected, that portion of the peripheral signal representing the taste stimulus is gone, unless compensatory reorganizational events are occurring in the remaining sensory receptors (an interesting but unexamined possibility). Thus, it is meaningful to identify the consequences of the signal removal on central gustatory processes, and if possible, relate them to neuroanatomical loci. Accordingly, such results can be compared with the behavioral effects of nerve transections potentially revealing the functional significance of the anatomical organization of the central gustatory system, caveats notwithstanding.

\section{Quinine-elicited FLI in the gNST: behavioral significance}

As discussed above, the quinine-induced FLI in the gNST in GLX rats was essentially the same as that observed in SHAM-W rats in the number, spatial pattern, and staining intensity of the labeled neurons. Therefore, if these quinine-related Fos-positive cells were behaviorally relevant, one might expect taste-related behavioral responses to quinine in GLX rats to approach those observed to water. Surprisingly, the effects of GL transection on taste-guided behavioral responses to quinine in the rat are unremarkable in comparison with its notable effect on $c$-fos expression. For example, previous reports have demonstrated that bilateral GL transection has no effect on quinine avoidance in two-bottle preference tests (Akaike et al., 1965; Grill et al., 1992) nor does it alter lick rate to quinine in brief-access tests across a wide array of suprathreshold quinine concentrations (Yamamoto and Asai, 1986; St. John et al., 1994). Although rats without presurgical experience with the stimulus have shown a statistically significant decrease in quinine avoidance, the extent of this decrease was modest (Markison et al., 1998), and rats with previous experience showed no deficit at all (St. John et al., 1994). Notably, performance in challenging behavioral tasks involving quinine as a taste stimulus, such as those that assess detection threshold (St. John and Spector, 1996) and taste discrimination (St. John and Spector, 1998) is not compromised by GL transection. Given that the facial nerve (in conjunction with the superior laryngeal nerve) can support quinine responsiveness in these behavioral paradigms, the results imply that these behaviors are not dependent on activity in the subpopulation of quinineresponsive FLI neurons, to the extent that the absence of FLI in a Fos-competent neuron is indicative of neural silence. It is perplexing that such a sizable population of FLI neurons that respond robustly to quinine stimulation may not substantially contribute to these taste-guided behaviors. Perhaps, these neurons are functionally active (receiving input from other nerves) when the GL is transected, but the level of activity in them, although not sufficient to stimulate Fos expression, can nonetheless support performance on these tasks.

There is one quinine-elicited behavior, however, that is severely compromised by GL transection: the gape, which is a stereotypical oromotor rejection response elicited by aversive stimuli. The number of gapes observed in response to intraoral quinine infusion is significantly reduced after transection of the GL but not after transection of the CT (Travers et al., 1987; Grill et al., 1992). The present data, which demonstrate GL transection to be more effective than $\mathrm{CT}$ transection in eliminating quinineinduced FLI in the gNST, compare favorably with this particular behavioral outcome. The degree of correspondence, however, must be tempered by the fact that GL transection did not completely abolish gaping to quinine; but interestingly, combined neurotomy (i.e., CTX + GLX) did (Grill and Schwartz, 1992).

Recently, DiNardo and Travers (1997) demonstrated in quinine-stimulated rats an impressively high correlation $(r=$ 0.90 ) between the number of FLI neurons in regions of the medullary reticular formation (RF) involved in oromotor reflex control and the number of gapes elicited. They found that the quinine-elicited FLI neurons in the RF intermingled substantially with afferent projections from the gNST. Accordingly, the authors suggested that the distinct pattern of FLI in the RF observed after quinine stimulation represents part of an "oral rejection circuit" involved in the control of behavioral reactions to aversive stimuli. The possibility exists that FLI in the gNST may simply be a reflection of feedback from a oral motor rejection circuit that is activated after infusion of an aversive substance such as quinine. If this is true then one would predict that other stimulus conditions, such as conditioned taste aversions (CTA), which elicit gapes and related rejection behaviors (Grill, 1985; Spector et al., 1988) would generate an identical distribution of FLI in the gustatory NST. Although CTA can increase FLI in the gustatory NST, the profile of results from studies conducted to date suggest that the pattern of quinine-induced FLI observed in our study cannot solely be explained by motor feedback (Swank and Bernstein, 1994; Houpt et al., 1996). Accordingly, it is intriguing to speculate that quinine stimulation induces FLI in the gNST because of its gustatory properties and may be preferentially expressed in those neurons involved in the afferent limb of an oromotor rejection circuit. 


\section{REFERENCES}

Akaike N, Hiji Y, Yamada K (1965) Taste preference and aversion in rats following denervation of the chorda tympani and the IXth nerve. Kumamoto Med J 18:108-109.

Andrew BL (1956) A functional analysis of the myelinated fibres of the superior laryngeal nerve of the rat. J Physiol (Lond) 133:420-432.

Beaver CJ, Mitchell DE, Robertson HA (1993) Immunohistochemical study of the pattern of rapid expression of $c$-fos protein in the visual cortex of dark-reared kittens following initial exposure to light. J Comp Neurol 333:469-484.

Beidler L (1969) Innervation of rat fungiform papilla. In: Olfaction and taste, III (Pfaffmann C, ed), pp 352-369. New York: Rockefeller UP.

Cleaton-Jones P (1976) A denervation study of taste buds in the soft palate of the albino rat. Arch Oral Biol 24:49-82.

Contreras RJ, Beckstead RM, Norgren R (1982) The central projections of the trigeminal, facial, glossopharyngeal and vagus nerves: an autoradiographic study in the rat. J Auton Nerv Syst 6:303-322.

Dahl M, Erickson RP, Simon SA (1997) Neural responses to bitter compounds in rats. Brain Res 756:22-34.

DiNardo LA, Travers JB (1997) Distribution of fos-like immunoreactivity in the medullary reticular formation of the rat after gustatory elicited ingestion and rejection behaviors. J Neurosci 17:3826-3839.

Dragunow M, Faull R (1989) The use of $c$-fos as a metabolic marker in neuronal pathway tracing. J Neurosci Methods 29:261-265.

Ehret G, Fischer R (1991) Neuronal activity and tonotopy in the auditory system visualized by c-fos gene expression. Brain Res 567:350-354.

Frank ME (1991) Taste-responsive neurons of the glossopharyngeal nerve of the rat. J Neurophysiol 65:1452-1463.

Frank ME, Contreras RJ, Hettinger TP (1983) Nerve fibers sensitive to ionic taste stimuli in the chorda tympani of the rat. J Neurophysiol 50:941-960.

Friauf E (1992) Tonotopic order in the adult and developing auditory system of the rat as shown by $c$-fos immunocytochemistry. Eur J Neurosci 4:798-812.

Friauf E (1995) C-fos immunocytochemical evidence for acoustic pathway mapping in rats. Behav Brain Res 66:217-224.

Ganchrow JR, Ganchrow D (1989) Long-term effects of gustatory neurectomy on fungiform papillae in the young rat. Anat Rec 225:224-231.

Grabauskas G, Bradley RM (1996) Synaptic interactions due to convergent input from gustatory afferent fibers in the rostral nucleus of the solitary tract. J Neurophysiol 76:2919-2927.

Grill HJ (1985) Physiological mechanisms in conditioned taste aversions. Ann NY Acad Sci 443:67-88.

Grill HJ, Norgren R (1978) The taste reactivity test. I. Mimetic responses to gustatory stimuli in neurologically normal rats. Brain Res 143:263-279.

Grill HJ, Schwartz GJ (1992) The contribution of gustatory nerve input to oral motor behavior and intake-based preference. II. Effects of combined chorda tympani and glossopharyngeal nerve section in the rat. Brain Res 573:105-113.

Grill HJ, Schwartz GJ, Travers JB (1992) The contribution of gustatory nerve input to oral motor behavior and intake-based preference. I. Effects of chorda tympani or glossopharyngeal nerve section in the rat. Brain Res 573:95-104.

Guth L (1957) Effects of glossopharyngeal nerve transection on the circumvallate papilla of the rat. Anat Rec 128:715-731.

Halsell CB, Travers SP, Travers JB (1996) Ascending and descending projections from the rostral nucleus of the solitary tract originate from separate neuronal populations. Neuroscience 72:185-197.

Hamilton RB, Norgren R (1984) Central projections of gustatory nerves in the rat. J Comp Neurol 222:560-577.

Harada S, Yamamoto T, Yamaguchi K, Kasahara Y (1997) Different characteristics of gustatory responses between the greater superficial petrosal and chorda tympani nerves in the rat. Chem Senses 22:133-140.

Harrer MI, Travers SP (1996) Topographic organization of Fos-like immunoreactivity in the rostral nucleus of the solitary tract evoked by gustatory stimulation with sucrose and quinine. Brain Res 711:125-137.

Houpt TA, Philopena JM, Joh TH, Smith GP (1996) c-Fos induction in the rat nucleus of the solitary tract by intraoral quinine infusion depends on prior contingent pairing of quinine and lithium chloride. Physiol Behav 60:1535-1541.

Jain N, Florence SL, Kaas JH (1998) Reorganization of somatosensory cortex after nerve and spinal cord injury. News Physiol Sci 13:143-149.

Kaczmarek L, Chaudhuri A (1997) Sensory regulation of immediateearly gene expression in mammalian visual cortex: implications for functional mapping and neural plasticity. Brain Res Rev 23:237-256.

King CT, Travers SP, Rowland NE, Lyons OL, Spector AC (1997) Gustatory nerve transection attenuates quinine-evoked Fos-like immunoreactivity in the rostral nucleus of the solitary tract. Chem Senses 22:719.

Lasiter PS (1992) Postnatal development of gustatory recipient zones within the nucleus of the solitary tract. Brain Res Bull 28:667-677.

Mack KJ, Mack PA (1992) Induction of transcription factors in somatosensory cortex after tactile stimulation. Mol Brain Res 12:141-147.

Markison S, St. John SJ, Spector AC (1999) Glossopharyngeal nerve transection reduces quinine avoidance in rats not given presurgical stimulus exposure. Physiol Behav 65:773-778.

Melzer P, Steiner H (1997) Stimulus-dependent expression of immediate-early genes in rat somatosensory cortex. J Comp Neurol 380:145-153.

Miller IJ (1977) Gustatory receptors of the palate. In: Food intake and chemical senses (Katsuki Y, Sato M, Takagi S Oomura Y, eds), pp 173-186. Tokyo: University of Tokyo.

Mitchell DE, Beaver CJ, Ritchie PJ (1995) A method to study changes in eye-related columns in the visual cortex of kittens during and following early periods of monocular deprivation. Can J Physiol Pharmacol 73:1352-1363.

Montero VM, Jian S (1995) Induction of c-fos protein by patterned visual stimulation in central visual pathways of the rat. Brain Res 690:189-199.

Morgan I, Curran T (1989) Stimulus-transcription coupling in neurons: role of cellular immediate-early genes. Trends Neurosci 12:459-462.

Morgan I, Curran T (1991) Stimulus-transcription coupling in the nervous system: Involvement of the inducible proto-oncogenes fos and jun. Annu Rev Neurosci 14:421-451.

Nejad MS (1986) The neural responses of the greater superficial petrosal nerve of the rat in response to chemical stimulation of the palate. Chem Senses 11:283-293.

Ogawa H, Hayama T (1984) Receptive fields of the solitarioparabrachial relay neurons with tongue afferent inputs in rats. Exp Brain Res 48:363-368.

Pfaffmann C (1955) Gustatory nerve impulses in rat, cat, and rabbit. J Neurophysiol 18:429-440.

Sagar SM, Sharp FR, Curran T (1988) Expression of $c$-fos protein in brain: metabolic mapping at the cellular level. Science 240:1328-1331.

Sharp FR, Sagar SM, Swanson RA (1993) Metabolic mapping with cellular resolution: $c$-fos vs. 2-deoxyglucose. Crit Rev Neurobiol 7:205-228.

Sheng M, Greenberg ME (1990) The regulation and function of c-fos and other immediate early genes in the nervous system. Neuron 4:477-485.

Shingai $T$ (1980) Water fibers in the superior laryngeal nerve of the rat. Jpn J Physiol 30:305-307.

Smith DV, Norman M, Shipley MT (1995) Are there CNS consequences of taste cell turnover? Chem Senses 18:631-632.

Spector AC, Breslin P, Grill HJ (1988) Taste reactivity as a dependent measure of the rapid formation of conditioned taste aversion: a tool for the neural analysis of taste-visceral associations. Behav Neurosci 102:942-952.

Steiner H, Gerfen CR (1994) Tactile sensory input regulates basal and apomorphine-induced immediate-early gene expression in rat barrel cortex. J Comp Neurol 344:297-304.

St. John SJ, Spector AC (1996) Combined glossopharyngeal and chorda tympani nerve transection elevates quinine detection thresholds in rats (Rattus norvegicus). Behav Neurosci 110:1456-1468.

St. John SJ, Spector AC (1998) Behavioral discrimination between quinine and $\mathrm{KCl}$ is dependent on input from the seventh cranial nerve: implications for the functional roles of the gustatory nerves in rats. J Neurosci 18:4353-4362.

St. John SJ, Garcea M, Spector AC (1994) Combined, but not single gustatory nerve transection substantially alters taste-guided licking behavior to quinine in rats. Behav Neurosci 108:131-140. 
St. John SJ, Markison S, Spector AC (1995) Salt discriminability is related to number of regenerated taste buds after chorda tympani nerve transection in rats. Am J Physiol 269:R141-R153.

Swank MW, Bernstein IL (1994) c-Fos induction in response to a conditioned stimulus after single trial taste aversion learning. Brain Res 636:202-208.

Travers JB, Grill HJ, Norgren R (1987) The effects of glossopharyngeal and chorda tympani nerve cuts on the ingestion and rejection of sapid stimuli: an electromyographic analysis in the rat. Behav Brain Res 25: 233-246.

Travers SP, Nicklas K (1990) Taste bud distribution in the rat pharynx and larynx. Anat Rec 227:373-379.

Travers SP, Norgren R (1995) Organization of orosensory responses in the nucleus of the solitary tract of rat. J Neurophysiol 73:2144-2162.

Travers SP, Pfaffmann C, Norgren R (1986) Convergence of lingual and palatal gustatory neural activity in the nucleus of the solitary tract. Brain Res 365:305-320.

Whitehead MC (1988) Neuronal architecture of the nucleus of the solitary tract in the hamster. J Comp Neurol 276:547-572.

Whitehead MC, Frank ME, Hettinger TP, Hou LT, Nah HD (1987) Persistence of taste buds in denervated fungiform papillae. Brain Res 405:192-195.

Whitehead MC, McGlathery ST, Manion BG (1995) Transganglionic degeneration in the gustatory system consequent to chorda tympani damage. Exp Neurol 132:239-250.

Yamada K (1966) Gustatory and thermal responses in the glossopharyngeal nerve of the rat. Jpn J Physiol 16:599-611.

Yamamoto T, Asai K (1986) Effects of gustatory deafferentation on ingestion of taste solutions as seen by licking behavior in rats. Physiol Behav 37:299-305. 